\title{
KIRSCHNER WIRE VERSUS TITANIUM ELASTIC NAILS IN PEDIATRIC FEMORAL SHAFT FRACTURES
}

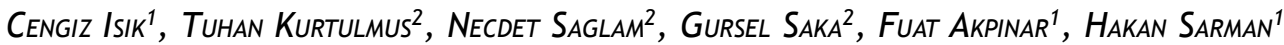

\section{ABSTRACT}

Objective: To evaluate the effectiveness of intramedullary fixation using the Kirschner-wire (K-wire) compared to the titanium elastic nail (TEN) in pediatric femoral shaft fractures. Methods: A sample of 42 pediatric patients with a mean age of $6.55 \pm 2.42$ years (range 4-11 years) presenting femoral shaft fractures underwent intramedullary fixation using the K-wire or TEN. Results: There was no significant difference found between groups, of which 16 (38.1\%) patients were treated with K-wire and 26 (61.9\%) patients were treated with TEN in terms of union duration. Moreover, none of the patients showed nonunion or a delayed union. Conclusions: The use of adjusted K-wire instead of TEN in the intramedullary fixation of femoral shaft fractures in selected children may be an advantageous surgical option due to the lower cost, easy accessibility and no need for a second surgery for implant removal. Level of Evidence III, Retrospective Study.

Keywords: Child. Bone nails. Femoral fractures. Humeral fractures. Fracture fixation, intramedullary. Titanium.

Citation: Isik C, Kurtulmus T, Saglam N, Saka G, Akpinar F, Sarman H. Kirschner wire versus titanium elastic nails in pediatric femoral shaft fractures. Acta Ortop Bras. [online] 2015;23(5):255-8. Available from URL: http://www.scielo.br/aob.

\section{INTRODUCTION}

The proximal femur, femoral shaft, or distal femur may be involved in femoral fractures in children, and an estimated annual incidence of pediatric femoral shaft fractures is 19 fractures per 100,000 children in the United States. ${ }^{1}$ Approximately $62 \%$ of all pediatric femoral fractures are femoral shaft fractures, ${ }^{1,2}$ with different surgical methods being performed successfully in the treatment of these fractures, including spica casts, plate fixation, bridge plates, external fixation, traction and intramedullary nailing. ${ }^{2-9}$

In recent years, intramedullary fixation methods have been preferred for pediatric femoral shaft fractures, particularly in patients over 5 years of age. The titanium elastic nail (TEN), locked intramedullary nail, or elastic stainless steel nail are among the most commonly used types of intramedullary fixation, ${ }^{10}$ with advantages in the ease of surgery, anatomical alignment, and for being a closed method of internal fixation. The disadvantages of TEN are implant migration into the joint or medulla, skin irritation, necessity of additional surgery for implant removal, and high cost.

Kirschner wire (K-wire) has been used for a long time in many different fields of orthopedic surgery, ${ }^{11}$ in particular, K-wire can be used for flexible nailing in pediatric fractures due to its easy availability and low cost. ${ }^{4}$
The aim of the present study is to analyze the results of pediatric femoral shaft fractures which were treated with intramedullary fixation using K-wire or TEN in terms of union, partial or total weight bearing, surgical waiting time, and implant removal.

\section{PATIENTS AND METHODS}

This retrospective study was performed at the Abant Izzet Baysal University Medical School Hospital. We evaluated 42 children with aged between 4-11 years who underwent intramedullary fixation due to femoral shaft fractures using K-wire or TEN (TST Tıbbi Aletler San. Ve Tic. Ltd. Şti. Maltepe, İstanbul, Turkey) between 2008 and 2011. Fractures treated with external fixation, plate-screw, or spica cast were excluded from the study.

All pediatric patients were operated under general anesthesia in supine positions. The use of TEN for femoral shaft fixation ( $n=26$ ) was performed using an incision of approximately $2 \mathrm{~cm}$ from medial and lateral to the level of the distal femoral metaphysis. After dissection of the soft tissue (to bone), an entrance was opened in the femur, with the aid of an awl, $2 \mathrm{~cm}$ superior to the medial and lateral distal femoral epiphyseal line. Then, a fracture reduction was performed under fluoroscopy and appropriate sized TENs chosen in accordance to femur medullary size were placed retrograde through the medial and lateral distal femur entrances. Two TENs were used in

All the authors declare that there is no potential conflict of interest referring to this article.

\footnotetext{
1. Abant Izzet Baysal University Medical School, Department of Orthopedics and Traumatology, Bolu, Turkey. 2. Umraniye Training and Research Hospital, Department of Orthopedics and Traumatology, Istanbul, Turkey.

This study was performed at the Abant Izzet Baysal University Medical School Hospital, Department of Orthopedics and Traumatology, Bolu, Turkey. Correspondence: Hakan Sarman, Abant Izzet Baysal University Medical Faculty, Department of Orthopedics and Traumatology. 14280, Gölköy, Bolu, Turkey. hakansarman@yahoo.com
} 
25 patients and three in one patient. TENs average size was $3.5 \mathrm{~mm}$ (2.5-4 mm). These implants were cut after passing 2 $\mathrm{mm}$ from the femoral cortex under the skin in all patients, and finally, the subcutaneous tissue and skin were closed.

A $2 \mathrm{~cm}$ incision was made over the level of the femoral distal metaphysis, from the medial and lateral sides, for those patients in whom the K-wire was used ( $n=16)$. Under fluoroscopy control, an entrance hole was created $2 \mathrm{~cm}$ proximal to the distal femoral epiphyseal level with the help of a drill. The K-wire was minimally inclined to form a " $\mathrm{C}$ "-shape, according to the fracture site. The end of the K-wire that was chosen in accordance with femur medullary size was blunted to prevent perforation of the across cortex, and was inclined at approximately $30^{\circ}$ to provide guidance. The K-wire was advanced to the fracture site using a hammer, at an approximate angle of $45^{\circ}$, with the help of a T-handle. This procedure was performed from both the medial and lateral sides, and each implant was advanced through the fracture site in the same direction. The fracture closed reduction was supported in all of the patients under fluoroscopy control, and the K-wires were advanced in the opposite directions after reaching the proximal fragments. The size of the K-wires used was $2.5 \mathrm{~mm}$. After stabilization and reduction control, the ends of the K-wires were leaved outside of the skin and were inclined. The wound was closed and dressing was applied.

Ankle and hip joint movements in patients were planned on the first postoperative day. Weight bearing was advised as much as the patient could tolerate, after considering the clinical and radiological findings.

Partial or full weight bearing was recommended according to clinical and radiological evaluations during the follow-ups, and in the event of a fracture callus or barely detected fracture site, the K-wires were removed (assuming that the union was sufficient) without a second surgery, since the ends of the K-wires were out of the skin. Among the patients who received the TEN procedure, after deciding radiologically and clinically that the union was sufficient (at least 6 months later), the implants were removed through the previous incisions in a second surgery. None of the patients were fixed or immobilized after the removal of the implants.

All patients were screened for fracture etiology, AO classification type, extent of displacement, additional pathology in the fracture zone, size and type of implant, as well as for the durations of the pre-operative period, follow-up, weight bearing, implant removal and union status.

NCSS (Number Cruncher Statistical System), 2007 PASS (Power Analysis and Sample Size), and 2008 Statistical Software (Utah, USA) were used for statistical analyses, and the data were shown as mean \pm standard deviation for the continuous variables, median (minimum - maximum) for ordinal variables, and frequency, with a percentage for categorical variables. The means were compared using the Student t or Mann-Whitney $U$ tests, where appropriate, and the qualitative data were compared using the Yates's Continuity Correction test. The results were evaluated at a significance level of $p<0.05$ or $p<0.01$.

\section{RESULTS}

A sample of 42 children ( 8 female, 34 male) with a mean age of $7.16 \pm 2.42$ (range, $4-11$ ) years were included in this study. Of these, 16 (38.1\%) patients were treated with K-wires and 26
(61.9\%) patients were treated with TEN. The mean age of the TEN group was $7.85 \pm 2.185$ (range, 4-11) years and K-wire group was $6.04 \pm 0.629$ (range, 4-7) years. The mean duration of follow-up was $24.88 \pm 10.61$ months for the TEN group and $29.00 \pm 5.02$ months for the K-wire group. The groups did not differ regarding age, gender and mean follow up-durations ( $p>0.05$ for all). The surgical waiting time was $3.31 \pm 3.26$ days for the TEN group and $0.94 \pm 0.44$ days for the K-wire group $(p<0.01)$. The demographic variables of the patients are summarized in Table 1.

The mean durations of the partial and full weight bearing stages were $41.27 \pm 7.80$ and $77.08 \pm 12.18$ days, respectively, for the TEN group, and 47.50 \pm 8.05 and $83.88 \pm 9.15$ days, respectively, for the K-wire group. The mean duration of partial weight bearing was significantly longer in the K-wire group than in the TEN group $(p=0.017)$, however, no significant difference was found between the groups for the mean full weight bearing duration $(p=0.058)$. There was pain during knee motion in $8(50 \%)$ patients with K-wires due to the irritation of the tensor fascia latae in the early postoperative period, although none of these patients reported pain or limited motion of the knee after K-wire removal. No significant difference was found between groups in terms of union duration, and none of the patients demonstrated nonunion or delayed union. The mean time before implant removal was 9.65 \pm 3.33 months (TEN group), and $3.19 \pm 0.25$ months (K-wire group). The time before the implant removal was significantly shorter in the K-wire group than in the TEN group ( $p=0.001)$. In K-wire group the implant was removed earlier than in TEN group, since the ends of K-wires were out of the skin, but the ends of the TEN were under the skin.

Within the TEN group, the etiological factors were out-of-vehicle traffic accidents (53.8\%), bicycle accidents (7.7\%), walking accidents (23\%), park accidents (3.3\%), sports accidents (3.8\%), and high falls (7.7\%). In the K-wire group, out-of-vehicle traffic accidents (43.8\%), in-vehicle traffic accidents (12.5\%), and high falls (43.8\%) were responsible for the injuries. One patient in the TEN group had a Type 1 open fracture, and one patient in the K-Wire group had a Type 2 open fracture, according to the Gustilo-Anderson classification. All other patients had closed fractures.

According to the AO classification, considering the TEN group, three of the fractures (11.5\%) were type 32-A1, four (15.4\%) were type 32-A2, 14 (53.8\%) were type 32-A3, and five (19.2\%) were type $32-\mathrm{B} 1$. For the K-wire group, two fractures (12.5\%) were type $32-\mathrm{A} 1$, two (12.5\%) were type 32-A2, and 12 (75\%) were type 32-A3.

While 22 patients (84.6\%) had closed reductions and four (14.4\%) had open reductions in the TEN group, all patients in the K-wire

Table 1. Demographic data of patients according to the groups.

\begin{tabular}{|c|c|c|c|c|}
\hline & \multicolumn{2}{|c|}{ Group } & \multirow[b]{2}{*}{$P$ value } \\
\hline & & $\begin{array}{c}\text { TEN } \\
(n=26)\end{array}$ & $\begin{array}{l}\text { K. wire } \\
(\mathrm{n}=16)\end{array}$ & \\
\hline \multicolumn{2}{|c|}{ Age (years old) } & $7.85 \pm 2.185$ & $6.04 \pm 0.629$ & ${ }^{a} 0,001^{* *}$ \\
\hline \multirow{2}{*}{ Gender } & Female & $5(19.2 \%)$ & $3(18.8 \%)$ & \multirow{2}{*}{${ }^{b} 1,000$} \\
\hline & Male & $21(80.8 \%)$ & $13(81.3 \%)$ & \\
\hline \multicolumn{2}{|c|}{ Postoperative follow-up (months) } & $24.88 \pm 10.61$ & $29.00 \pm 5.02$ & ${ }^{\mathrm{a}} 0,099$ \\
\hline \multicolumn{2}{|c|}{ Surgery waiting time (days) } & $3.31 \pm 3.26(2.00)$ & $0.94 \pm 0.44(1.00)$ & ${ }^{\circ} 0,001^{\star \star}$ \\
\hline
\end{tabular}


group had closed reductions. None of the patients showed implant migrations, or joint irritation. Four patients in the K-wire group who have grade 1 pin-tract infections (according to The Checketts-Otterburn's grading system for level of pin-site infection). Therefore, pin site care was improved to avoid infections. One patient in the K-wire group who developed a femur shaft fracture due to a high fall had a simple bone cyst in the fracture area. In this patient, fracture healing was observed, however the simple bone cyst did not improve, and this patient's parents did not approve surgery for removing the cyst. Figure $1 \mathrm{~A}-\mathrm{F}$ shows the radiograms of that patient. Table 2 depicts the Gustilo-Anderson and AO classifications of the fractures, etiological factors, reduction types, and additional pathologies, comparing the two groups.

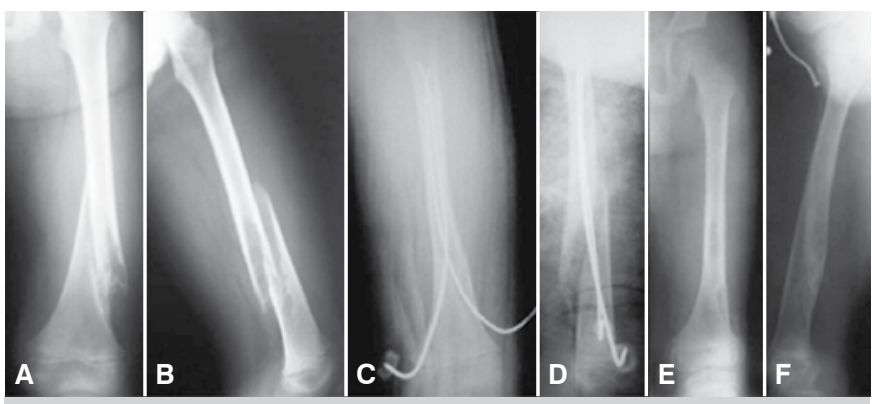

Figure 1. (A, B): 6 year old male patients presented with distal 1/3 femur oblique spiral fracture occurred on the region of simple bone cyst. Preoperative antero-posterior and lateral $X$-ray view. (C, D): First postoperative week antero-posterior and lateral x-ray view. $(E, F)$ : Third year postoperative antero-posterior and lateral X-ray view.

Table 2. Gustilo-Anderson open fracture classification, etiology, AO classification and reduction properties of the patients according to the groups.

\begin{tabular}{c|c|c|c}
\hline \multicolumn{2}{c|}{} & TEN (n=26) & K-wire (n=16) \\
\cline { 3 - 4 } \multicolumn{2}{c|}{} & $\mathbf{n}(\%)$ & $\mathbf{n}(\%)$ \\
\hline \multirow{3}{*}{$\begin{array}{c}\text { Gustilo-Anderson } \\
\text { classification }\end{array}$} & Closed fracture & $25(96.2 \%)$ & $15(93.8 \%)$ \\
\cline { 2 - 4 } & Grade I & $1(3.8 \%)$ & $0(0.0 \%)$ \\
\cline { 2 - 4 } & Grade II & $0(0.0 \%)$ & $1(6.3 \%)$ \\
\hline \multirow{4}{*}{ Etiology } & Motor vehicle accident & $14(53.8 \%)$ & $9(56.3 \%)$ \\
\cline { 2 - 4 } & Bicycle accident & $2(7.7 \%)$ & $0(0.0 \%)$ \\
\cline { 2 - 4 } & Sports & $8(30.7 \%)$ & $0(0.0 \%)$ \\
\cline { 2 - 4 } & Fall from height & $2(7.7 \%)$ & $7(43.8 \%)$ \\
\hline \multirow{4}{*}{ AO classification } & A1 & $3(11.5 \%)$ & $2(12.5 \%)$ \\
\cline { 2 - 4 } & A2 & $4(15.4 \%)$ & $2(12.5 \%)$ \\
\cline { 2 - 4 } & A3 & $14(53.8 \%)$ & $12(75.0 \%)$ \\
\cline { 2 - 4 } & B1 & $5(19.2 \%)$ & $0(0.0 \%)$ \\
\hline \multirow{2}{*}{ Reduction type } & Closed & $22(84.6 \%)$ & $16(100.0 \%)$ \\
\cline { 2 - 4 } & Open & $4(15.4 \%)$ & $0(0.0 \%)$ \\
\hline
\end{tabular}

\section{DISCUSSION}

The treatment of femoral shaft fractures in children depends on the age and weight of the patient, and the type of fracture. For patients under five years of age, the treatment options are generally conservative (e.g. pelvic bandage, pelvipedal casting, or pelvipedal casting after skeletal traction). Above five years of age, external fixation, stabilization using plate screws or intramedullary fixation are used in the treatment of femoral shaft fractures. ${ }^{6,12,13}$ In the present study, procedures with
K-wire and TEN were found to be equally effective in the surgical treatment of pediatric femoral shaft fractures. The low cost, easy accessibility and no need for a second surgery are the main advantages of using K-wire, as opposed to TEN.

Valgus deformities in the proximal femur, narrowing of the femur neck, and trochanteric physeal arrest complications have been reported for antegrade intramedullary fixation through the trochanter tip entry. ${ }^{7-9}$ In addition, Carey and Galpin have reported antegrade administration of flexible intramedullary nailing in 25 patients and observed growth arrest in the greater trochanter apophysis of the femur. ${ }^{3}$ It has been reported in some biomechanical studies that retrograde fixation provided more strength than antegrade nailing against the torsional and bending forces in mid-femoral shaft fractures. ${ }^{14,15}$ We performed retrograde intramedullary fixation techniques in all patients, and none of our patients required intervention to correct a reduction loss, and none of the K-wires showed deformation outside of the acceptable limits.

Flynn et al. performed traction and casting in 35 children, and intramedullary TEN in 49 children with femoral shaft fractures, ${ }^{16}$ and found that the time periods of hospitalization, standing, and return to school were lower in patients treated with TEN., The hospital cost, however, did not differ between the treatment groups. Although we did not evaluate the length of hospitalization in the present study, our clinical experience shows that pediatric femoral shaft fractures treated with K-wire have shorter hospitalization times when compared to TEN, possibly due to early surgical interventions in the K-wire group, since K-wire is available every time in the orthopedics surgical room, which does not occur with TEN. Ozdemir et al. ${ }^{17}$ reported that pelvic and thigh brace applications at the first day postoperative day after flexible steel nailing for the treatment of pediatric femoral shaft fractures provided earlier motion and weight bearing. However, we did not apply braces in our patients. In the case of insufficient stabilization, we applied a plaster splint, which was removed after 2-3 weeks to increase the patient's mobility.

Recently, the use of intramedullary elastic Ender-type nails and titanium nails for the fixation of pediatric femoral fractures has gained popularity, ${ }^{16}$ although some authors have reported the successful use of K-wires for intramedullary fixation due to their lower cost and availability. ${ }^{4,6,18-20}$ In the present study, we used K-wires in 16 children and TEN in 26 children for intramedullary fixation. The surgical waiting time for the TEN group was significantly higher when compared to the K-wire group due to the fact that TEN implant are not routinely kept in the hospital. Additionally (different from the current literature), we left the distal tilted end of the K-wire outside of the skin. The advantages of the placement of the distal ends of the K-wires outside of the skin are that implant removal does not require further surgery, prevention of bone medullary migration, and prevention of joint irritation due to the implant. The disadvantages may include pin tract infections and pain during knee motion due to tensor fascia latae irritation; however, we did not observe deep pin tract infections in our study population. Knee motions were painful and restricted in all patients in the K-wire group due to the irritation of the fascia latae during the early postoperative period. After the removal of the K-wire, the painful and limited knee motions resolved spontaneously. In the present study, all implanted K-wires were removed under sedation without additional surgical intervention, and none of the patients developed 
infections. In the TEN group, all patients required incisions and soft tissue dissection for removal of the implants. The AAOS study group did not make any recommendations whether or not to remove the implants in the asymptomatic femoral diaphyseal fractures. ${ }^{13}$ Flynn et al. reported that if the fracture line cannot be seen radiologically, and a sufficient callus has formed, the implants can be removed in the following circumstances: loss of range of motion, presence of knee effusion, and soft tissue irritation or pain due to the implant itself. ${ }^{16}$

The relatively small sample size of both groups is the main limitations of the present study. Further prospective studies are necessary to evaluate the effectiveness of K-wire and TEN in pediatric femoral shaft fractures with similar age groups using a larger sample.

\section{CONCLUSION}

In this study, we observed no different in term of union, partial or total weight bearing in both groups. The use of the adjusted
K-wire instead of TEN in the intramedullary fixation of femoral shaft fractures in children may be an advantageous surgical option in terms of lower cost, quick hospital discharge, material supply, and surgical waiting time. Moreover, the distal end of the K-wire which is left out of the skin does not require surgical removal, and may prevent the complications of joint and/or medullary migration. However, the pin-tract infections could represent severe complication.

\section{AUTHOR CONTRIBUTIONS}

This manuscript, which is a multi-institutional study, has six authors. Each author individually and significantly contributed to the development of the manuscript. $\mathrm{Cl}$ and $\mathrm{HS}$ were the major contributors in writing the manuscript. Cl, TK NS and GS performed the surgery, followed the patients and gathered the clinical data. FA and HS evaluated data of statistical analysis. $\mathrm{Cl}$, FA and HS performed the literature search, reviewed the manuscript and contributed to the intellectual concept.

\section{REFERENCES}

1. Rewers A, Hedegaard H, Lezotte D, Meng K, Battan FK, Emery K, et al. Childhood femur fractures, associated injuries, and sociodemographic risk factors: a populationbased study. Pediatrics. 2005 May;115(5):e543-52.

2. Sarman H, Işık C, Inanmaz ME, Köse KÇ, Tekelioğlu ÜY, Boraz I, et al. Postoperative fracture complications seen in the patients after sitting imbalance correction. Acta Medica Anatolia. 2014;2(3):108-10.

3. Carey TP, Galpin RD. Flexible intramedullary nail fixation of pediatric femoral fractures. Clin Orthop Relat Res. 1996 Nov(332):110-8.

4. Chitgopkar SD. Flexible nailing of fractures in children using stainless steel Kirschner wires. Journal of pediatric orthopaedics B. 2008;17(5):251-55.

5. Lopes JI, Rotoly AL, Dos Santos CA, Cardoso FJ. New method of preoperative immobilization for the proximal femoral fractures. Acta Ortop Bras. 2013 Jan;21(1):40-2.

6. Matsubara H, Yasutake H, Matsuda E, Uehara K, Niwada M, Tanzawa Y. Treatment of femoral shaft fractures in children using intramedullary Kirschner wire pinning. Journal of Orthopaedic Science. 2005;10(2):187-91

7. Gonzalez-Herranz P, Burgos-Flores J, Rapariz JM, Lopez-Mondejar JA, Ocete $J G$, Amaya S. Intramedullary nailing of the femur in children. Effects on its proximal end. J Bone Joint Surg Br. 1995 Mar;77(2):262-6.

8. Keeler KA, Dart B, Luhmann SJ, Schoenecker PL, Ortman MR, Dobbs MB, et al. Antegrade intramedullary nailing of pediatric femoral fractures using an interlocking pediatric femoral nail and a lateral trochanteric entry point. J Pediatr Orthop. 2009 Jun;29(4):345-51.

9. Raney EM, Ogden JA, Grogan DP. Premature greater trochanteric epiphysiodesis secondary to intramedullary femoral rodding. J Pediatr Orthop. $1993 \mathrm{Jul}-$ Aug;13(4):516-20.

10. Stans AA, Morrissy RT, Renwick SE. Femoral shaft fracture treatment in patients age 6 to 16 years. Journal of Pediatric Orthopaedics. 1999;19(2):222-28.

11. Firoozabadi R, Kramer PA, Benirschke SK. Kirschner wire bending. J Orthop Trauma. 2013 Nov;27(11):e260-3.

12. Poolman RW, Kocher MS, Bhandari M. Pediatric femoral fractures: a systematic review of 2422 cases. J Orthop Trauma. 2006 Oct;20(9):648-54.

13. Kocher MS, Sink EL, Blasier RD, Luhmann SJ, Mehlman CT, Scher DM, et al Treatment of pediatric diaphyseal femur fractures. J Am Acad Orthop Surg. 2009 Nov; 17(11):718-25

14. Fricka KB, Mahar AT, Lee SS, Newton PO. Biomechanical analysis of antegrade and retrograde flexible intramedullary nail fixation of pediatric femoral fractures using a synthetic bone model. J Pediatr Orthop. 2004 Mar-Apr;24(2):167-71.

15. Mehlman CT, Nemeth NM, Glos DL. Antegrade versus retrograde titanium elastic nail fixation of pediatric distal-third femoral-shaft fractures: a mechanical study. J Orthop Trauma. 2006 Oct;20(9):608-12.

16. Flynn JM, Luedtke LM, Ganley TJ, Dawson J, Davidson RS, Dormans JP, et al. Comparison of titanium elastic nails with traction and a spica cast to treat femoral fractures in children. J Bone Joint Surg Am. 2004 Apr;86-A(4):770-7.

17. Ozdemir HM, Yensel U, Senaran H, Mutlu M, Kutlu A. Immediate percutaneous intramedullary fixation and functional bracing for the treatment of pediatric femoral shaft fracture. J Pediatr Orthop. 2003 Jul-Aug;23(4):453-7.

18. Al-Zahrani S, Al-Fahel H, Zamzam M, IKRAM MA, Kremli M, Ali A, et al. Treatment of proximal third femoral shaft fractures in children by intramedullary Kirschner wires. Saudi medical journal. 1998;19(1):41-44

19. Jawadi $A H$, Abdul-Samad A. Intramedullary Kirschner wire (K-wire) fixation of femoral fracture in children. J Child Orthop. 2007 Nov; 1(5):277-80.

20. Qidwai SA, Khattak ZK. Treatment of femoral shaft fractures in children by intramedullary Kirschner wires. J Trauma. 2000 Feb;48(2):256-9. 